\title{
A IMPORTÂNCIA DO EXAME PROCTOLÓGICO
}

\author{
Vinicius Machado do Couto Soares ${ }^{1}$
}

RESUMO: A proctologia é o ramo especializado da cirurgia geral que estuda as doenças anorretais. Numerosos problemas anorretais podem ser diagnosticados simplesmente pela inspeção cuidadosa da área perianal. O exame de proctologia é imprescindível na presença de sintomas decorrentes de problemas intestinais. $O$ objetivo deste trabalho é enfatizar, por meio de uma revisão de literatura, a importância do exame físico proctológico, como meio preventivo de doenças anorretais. $\mathrm{O}$ resultados mostraram o quão é importante a realização de exames físicos relacionados ao trato gastrointestinal no controle, combate e prevenção de doenças anorretais.

Palavras-chave: Exame Físico. Doenças Anorretais. Neoplasias. Exame Proctológico.

ABSTRACT: Proctology is the specialized branch of general surgery that studies anorectal diseases. Numerous anorectal problems can be diagnosed simply by carefully inspecting the perianal area. Proctology examination is essential in the presence of symptoms resulting from intestinal problems. The objective of this work is to emphasize, through a literature review, the importance of proctological physical examination as a preventive means of anorectal diseases. The results showed how important it is to perform physical examinations related to the gastrointestinal tract in the control, combat and prevention of anorectal diseases.

Keywords: Physical Examination. Anorectal Diseases. Neoplasms. Proctological Examination.

\section{INTRODUÇÃO}

Como afirma McCrae (1870-1935) "faz-se mais falta por não olhar do que por não saber.” Esta declaração sublinha a importância primordial do exame clínico no diagnóstico proctológico. Numerosos problemas anorretais podem ser diagnosticados simplesmente pela inspeção cuidadosa da área perianal. Embora uma consulta proctológica possa representar uma atividade profissional de rotina para o médico, é preciso lembrar que para o paciente é uma questão bem diferente.

O exame de proctologia é imprescindível na presença de sintomas decorrentes de problemas intestinais. Muitas pessoas ficam assustadas com a ideia de uma visita ao proctologista e tendem a adiá-la. Constrangimento, medo e desinformação podem

r Médico, residente de Cirurgia Geral da Secretaria Municipal de Saúde de Macaé-RJ. Email:vmiplzon@gmail.com 
fazer o indivíduo ignorar os sintomas de doenças que às vezes podem ser muito graves. Além disso, mesmo doenças facilmente tratáveis podem piorar e se tornar crônicas se não forem tratadas (DE ARAÚJO, 2017).

O exame retal é um exame íntimo e às vezes desconfortável que é feito com mais frequência quando há suspeita de doença (geralmente doença gastrointestinal ou geniturinária) ou já identificada. Também pode ser feito como parte de um exame de triagem quando não há suspeita ou expectativa de doença, mas o exame é realizado como parte de um processo de triagem completo (MUCABREL, RAMOS \& DUQUE, 2020).

O toque retal é frequentemente necessário em pacientes idosos porque os sintomas e a doença surgem com mais frequência em pacientes idosos, entretanto este exame é suma importância no diagnóstico preventivo de doenças do trato gastrointestinal, como o câncer colorretal (FERREIRA \& LIMA, 20I9).

\section{METODOLOGIA}

Para elaboração deste estudo foi realizada uma revisão da literatura sobre Câncer colorretal, foi realizada uma pesquisa através de sites de busca acadêmica: PubMed, Lilacs e Scielo, durante o período de 2015 a 2021. utilizando como termos descritores: câncer, proctologia, doença anorretal, doenças colorretal, câncer colorretal, exame físico, neoplasia intestinal, associando-os em diferentes combinações utilizando a ferramenta Mesh. Foram utilizados os artigos escritos em português e inglês para compor o escopo do trabalho.

\section{CONTEÚDO DA REVISÃO}

A proctologia é o ramo especializado da cirurgia geral que estuda as doenças anorretais. Distúrbios anorretais são condições comuns. Sua prevalência na população geral é provavelmente muito maior do que a observada na prática clínica, pois a maioria dos pacientes não procura atendimento médico. Não há prevalência de sexo, homens e mulheres de qualquer idade podem ser acometidos. O espectro de distúrbios anorretais varia de benigno a potencialmente fatal (câncer anorretal) (NETO et al., 202I). 
Os sintomas muitas vezes não são específicos e difíceis de avaliar; restrições culturais e sociais tornam difícil para alguns pacientes falar sobre problemas em distúrbios anais, e os médicos nem sempre perguntam aos pacientes sobre possíveis sintomas, o que pode atrasar o diagnóstico. Na Figura I tem-se a divisão das doenças anorretais classificadas segundo Swarowsky et al. (2021).

Figura I- Divisão das principais doenças anorretais segundo critérios de incidência.

\begin{tabular}{|c|c|c|}
\hline Comum & Menos comum & Incomum \\
\hline $\begin{array}{l}\text { Hemorroidas } \\
\text { Fissuras } \\
\text { anais Fístulas anais } \\
\text { Abscessos } \\
\text { Pólipos } \\
\text { Prolapso retal Pólos } \\
\text { cutâneos anais ou pilha sentinela } \\
\text { Sepse anorretal }\end{array}$ & $\begin{array}{l}\text { Doença do seio pilonidal } \\
\text { Neoplasia } \\
\text { Condilomas } \\
\text { Massas de tecido conjuntivo } \\
\text { Antibioma (abscesso organizado) } \\
\text { Condições } \\
\text { inflamatórias Doenças inflamatórias intestinais } \\
\text { Papilas anais hipertrofiadas }\end{array}$ & $\begin{array}{l}\text { Estenoses do canal anal ou reto } \\
\text { Úlcera retal solitária } \\
\text { Incontinência }\end{array}$ \\
\hline
\end{tabular}

Fonte: Swarowsky et al. (2021).

A avaliação dos distúrbios anorretais compreende uma história e exame físico cuidadosos antes que o paciente possa ser submetido a várias investigações. Esses distúrbios são comumente encontrados na prática cirúrgica geral, e os pacientes geralmente estão com dor, muitas vezes ansiosos e frequentemente constrangidos com o exame. A detecção cuidadosa de sintomas anorretais e gastrointestinais (GI) e a presença de doença sistêmica esclarecem o diagnóstico da maioria dos distúrbios anorretais. É útil fazer perguntas padrão ao paciente sobre o tempo $e$ as circunstâncias do início dos sintomas, duração, qualidade e eventual exposição à radiação (AZEVEDO \& HORTA, 2018).

Alterações nos hábitos intestinais devem ser observadas: alterações na cor, frequência ou consistência das fezes e presença de esforço, flatulência e incontinência de fezes sólidas ou líquidas. A presença de doença de Crohn, câncer, e pólipos podem favorecer o aparecimento de formas incomuns de problemas anorretais. No entanto, também doenças sistêmicas, como síndrome da imunodeficiência adquirida, 
malignidades gastrointestinais, diabetes mellitus e coagulopatia, são propensas a desenvolver complicações mais graves das condições anorretais padrão (DE SANTANA, SINATORA \& DE ARGOLLO, 2017).

Os pacientes devem fornecer informações diretamente ao médico examinador sobre as práticas sexuais envolvendo o ânus. $O$ exame físico deve ser feito em privado, respeitando a modéstia do paciente. $O$ paciente pode então relaxar o esfíncter externo para facilitar um exame completo. A escolha da posição depende do equipamento disponível, da preferência e experiência do examinador e do habitus do paciente. As posições mais frequentes para exames proctológicos são três: litotômica, joelho-cotovelo e lateral esquerda; a última costuma ser a mais utilizada na cirurgia (AQUINO et al., 202I).

Assim, a história e o exame físico podem levar ao diagnóstico da lesão anal e podem excluir o carcinoma marginal clássico, que apresenta aspecto de couve-flor ou como uma úlcera extensa com bordas muito espessas. No entanto, todas as formas clínicas são possíveis e uma biópsia é sem dúvida indicada (PINTO et al., 202I).

\section{CONSIDERAÇÕES FINAIS}

A proctologia nasceu tardiamente e desenvolveu-se lentamente ao longo dos anos. No entanto, nos últimos 20 anos, um interesse renovado tem se afirmado, graças à contribuição de muitos autores. Assim, pela simplicidade de execução e alta sensibilidade diagnóstica do exame físico proctológico, destacamos a importância da sua realização por qualquer profissional médico no que se refere ao combate, prevenção ou diagnóstico de doenças anorretais.

\section{REFERÊNCIAS}

DE ARAÚJO, José Juvenal. A IMPORTÂNCIA DO EXAME PROCTOLÓGICO NO ESTADIAMENTO DO CÂNCER DO COLO UTERINO ERIVALDO FERNANDES LIRA-ASBCP MANUEL DA SILVA RESENDE-TSBCP MURILO BOAVISTA PESSOA MENDES-TSBCP. 2017.

MUCARBEL, Igor Murilo Gomes; RAMOS, Tyelle Joyce Leite; DUQUE, Marcos André Araújo. A importância do exame psa-antígeno prostático específico-para a prevenção do câncer de próstata. Brazilian Journal of Development, v. 6, n. I2, p. 94184-94195, 2020. 
FERREIRA, Jamily Brígido; LIMA, Sebastiana Shirley de Oliveira. Exame de toque retal como prevenção ao câncer de próstata: uma revisão de literatura. 2019.

NETO, I. J. F. C. et al. A Importância da Manometria Anorretal na Propedêutica Pré-operatória em Pacientes com Fissura Anal Crônica. Journal of Coloproctology, v. $4 \mathrm{I}, \mathrm{n}$. S oI, p. Ar86, 202I.

SWAROWSKY, I. L. et al. Diagnóstico Diferencial da Proctalgia Aguda: Uma Revisão Sistemática. Journal of Coloproctology, v. 4I, n. S or, p. Ar87, 202I.

AZEVEDO, Ana; HORTA, David. Abcessos e fistulas perianais-Recomendações Perianal supurations-Recommendations. 2018.

DE SANTANA, Marcela Amaro; SINATORA, Julia Bazzo; DE ARGOLlO, Ricardo. FÍSTULA RETOVESTIBULAR COM ÂNUS PÉRVIO: RELATO DE CASO E REVISÃO DA LITERATURA. 2017.

AQUINO, A. C. et al. Adenocarcinoma Mucinoso em Fístula Anorretal Recidivada: Relato de Caso. Journal of Coloproctology, v. 4I, n. S or, p. A035, 2021.

PINTO, Rodrigo Ambar et al. A experiência do médico examinador tem influência na avaliação do tônus anal em comparação à manometria anorretal?. Arquivos de Gastroenterologia, v. 56, n. I, p. 79-83, 2019. 